\title{
Biometric Recognition of Newborns: Identification using Palmprints
}

\author{
Rubisley P. Lemes, Olga R. P. Bellon, Luciano Silva* \\ Universidade Federal do Paraná - Curitiba, Paraná, Brazil \\ IMAGO Research Group - www.imago.ufpr.br \\ \{rubisley, olga, luciano\}einf.ufpr.br
}

\author{
Anil K. Jain \\ Michigan State University \\ East Lansing, Michigan \\ jain@cse.msu.edu
}

\begin{abstract}
We present some results on newborn identification through high-resolution images of palmar surfaces. To our knowledge, there is no biometric system currently available that can be effectively used for newborn identification. The manual procedure of capturing inked footprints in practice for this purpose is limited for use inside hospitals and is not an effective solution for identification purposes. The use of friction ridge patterns on the hands of newborns is challenging due to both the small size of newborn's papillary ridges, which are, on average, 2.5 to 3 times smaller than the ridges in adult fingerprints, and their fragility, making them amenable to deformation. The proposed palmprint based automatic system for newborn identification is relatively easy to use and shows the feasibility of this approach. Experiments were performed on images collected from 250 newborns at the University Hospital (Universidade Federal do Paraná). An image acquisition protocol was developed in order to collect suitable images. When considering the good quality palmar images, the results show that the proposed approach is promising.
\end{abstract}

\section{Introduction}

Newborn identification is crucial to avoid baby swaps in hospitals, child kidnapping and illegal adoptions. As an example, statistics show that among the millions of births that occur annually in Brazil there is one baby swap for every 6,000 births. One of the main concerns of the mothers during the child birth has to do with newborn swaps.

The identification of newborns is practiced worldwide through the acquisition of footprints using ink and paper method and the use of bracelets. DNA testing is an alternative identification technique, but it is both expensive and time consuming.

* The authors would like to thank Conselho Nacional de Desenvolvimento Científico e Tecnológico (CNPq) and Brazilian Ministry of Education.
According to [11], even with well-trained staff, good equipment and appropriate techniques, it is very difficult to collect good quality palmprints or footprints from newborns, mainly because the skin is covered with an oily substance and due to the extreme fragility of the ridges. It is generally agreed that footprints as currently captured (ink and paper) are not useful for identification purposes. Further, the results in [11] show that palmprints yield better quality images than footprints despite being more difficult to acquire, since newborn babies do not willingly open their hands.

Despite the maturity of biometrics technology, there is no biometric system, to the best of our knowledge, that has been developed for newborn identification purposes. The commercial biometric systems that are used for adult identification [1,9], are not feasible when applied to newborns, since biometric features (e.g., fingerprint and iris) are difficult to capture for babies. Another limitation is that most biometric traits, for example face, undergo change during child growth, making the collected data worthless for later identification.

One of the most well-known and commonly used biometric technology is based on friction ridge patterns. The papillary ridges on the fingers, palms and soles of the human embryo are completely formed after the 18th week of gestation [3]. So, in principle, it should be possible to collect dactyloscopic information of all newborns. However, newborns' papillary ridges are, on average, 2.5 to 3 times smaller than in adults, very fragile, and easily deformed upon contact. According to initial estimates presented in [11], fingerprint images of newborns must be acquired at 1500ppi resolution to clearly observe their ridges.

This paper presents a new approach to the challenging problem of newborn identification. Our work is based on high-resolution images of the palm dactyloscopic information and its main contribution is the development of appropriate techniques for acquiring, processing and recognizing these challenging images. In our experiments, we collected newborn images within 48 hours after the birth. The results show that our methodology based on palmprints can 
be used for comparison purposes in case of doubt about the baby identity, provided the images are carefully acquired to ensure their resolution and quality.

\section{Image Acquisition}

We propose a methodology for newborn identification based on high-resolution palmprint images. To conduct preliminary studies with the objective of developing an automatic system for newborn identification, we used a commercially available sensor (CrossMatch LSCAN 1000P) that best matched our needs. Despite the main disadvantage of providing only 1000ppi images instead of the required 1500ppi, the Crossmatch sensor is not ergonomically suitable for newborns either, making it difficult to acquire palmar images. However, we found that a subset of the acquired images is indeed suitable for identification.

We collected 1,221 palmprints $(4964 \times 5120$ pixels, 256 gray levels, 1000ppi) from 250 newborns at the University Hospital (Universidade Federal do Paraná) to create a database (NB_ID). All images were acquired between one to 48 hours after birth. We set up to five image acquisition sessions per baby. In each session, three palmprint images from the right palm were collected. In order to automatically assess the quality of the images in the database, we use the method proposed in [12] to classify the images into five levels: (1) good, (2) normal, (3) dry, (4) wet and (5) spoiled (Fig. 1). The quality assessment results showed that only $5 \%$ of palmprint images had sufficient quality (e.g., images classified as good) to satisfy the requirements for automatic matching and recognition. The main reasons for this low rate of images classified as good or normal are the low sensor resolution(1000ppi), and difficulties in holding the newborn's palmar surface at the correct position and pressure (to avoid ridge deformation). We also classify the images manually into these five quality levels and the manual classification also showed that only $4.3 \%$ of palmprints images were classified as good.

After testing a variety of images for recognition purposes, we concluded that only images classified as "good" are useful to ensure the identity of the child before he/she leaves the hospital.

In order to develop a suitable image acquisition protocol to obtain good images, the first step is to appropriately prepare the palmprint of newborns to remove the oily substance and generate a clean surface. To this end, the images were collected after using different cleaning techniques (e.g., only soap and water, only alcohol (Hidrated Ethanol with alcoholic graduation of 70\%) or both). In addition, we performed experiments regarding pressure (e.g., high or low) and exposure time (e.g., one second of acquisition or approximately four seconds using image preprocessing techniques during the acquisition) in an effort to acquire good quality images.

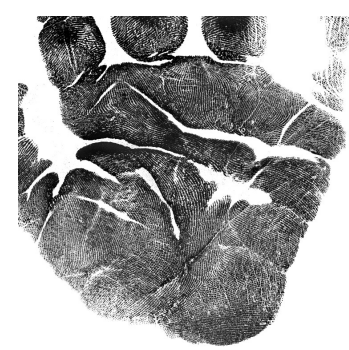

(a)

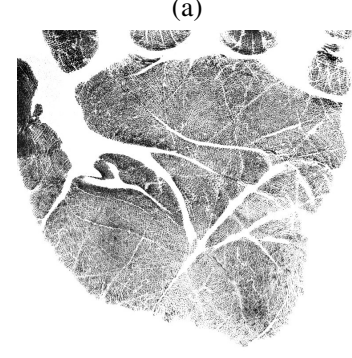

(c)

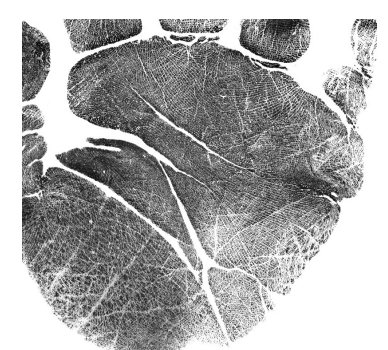

(b)

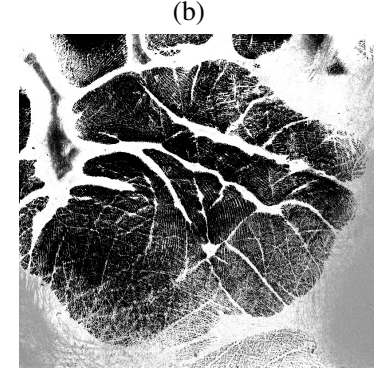

(d)

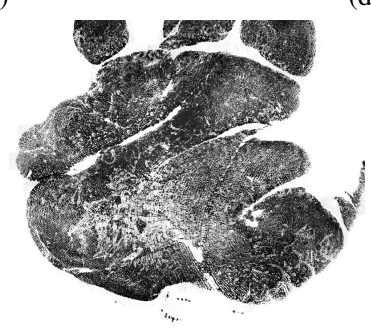

(e)

Figure 1. Image classification based on quality: (a) good, (b) normal, (c) dry, (d) wet, (e) spoiled.

As indicated earlier, a large fraction of collected images are not suitable for identification. Besides the fact that the 1000ppi sensor resolution is not adequate for newborns, there are some additional reasons for poor quality images: (1) during the scanning process, the babies move their foot/hand, leading to spoiled images or ridges deformation; (2) the pressure applied on the foot/hand is too high, causing ridges deformation or too low, resulting in non-visible ridges; (3) the skin is very dry when only alcohol is used for cleaning, even though, the alcohol provides good skin cleansing; (4) the oily substance on the palm is not completely removed when only soap and water is used and the skin is too wet if not properly dried. To collect good quality images, we concluded that the best protocol should be as follows: (1) it is necessary to use alcohol as the first cleaning step; (2) to ensure good skin cleansing, a second cleaning step must be applied, using soap and water; (3) the hand needs to be dried using paper towel; (4) it is very important to have a fast exposure time and to apply the right pressure of the palm on the sensor surface. 


\section{Image Preprocessing}

The proposed palmprint image processing framework consists of the following stages: (1) Minimum Bounder Rectangle (MBR) definition, (2) orientation correction, (3) finger removal, (4) Region of Interest (ROI) segmentation, (5) image classification and equalization [12], (6) Short Time Fourier Transform [2], (7) directional field (DF) estimation, (8) frequency estimation, (9) recoverable ridges detection, (10) Gabor Filtering and (11) delta point(s) detection.

In stage 1, the MBR is detected to cut down the computational burden in the subsequent stages. The image size is reduced, on average, from $4964 \times 5120$ to $1600 \times 1500$. In stage 2 , we automatically estimate a rotation angle to rectify the image orientation, similar to the procedure in [10]. In the finger removal stage, points $b$ and $c$ are used to divide the palmprint into 3 areas of equal size (Fig. 2a) to define point $e$ (new origin) similar to [10]. At the origin, we fit a parabola that least intercepts the palmprint, which is used to remove the fingers from the captured image (Fig. 2b green parabola). The first point on the palmprint is found in each column inside of a1. Point $f$ is the lowest point among all the detected points. The whole image below and to the left of $f$ is then cropped (Fig. 2c). This preprocessing stage is necessary to extract the palmprint ROI just below the fingers.

Stage 5 improves the image quality based on [12]. The image is locally equalized through the technique known as Contrast Limited Adaptive Histogram Equalization (CLAHE) [13] (Fig. 2c), which overcomes the limitations of the standard histogram equalization (HE).

An image enhancement operation based on the Short Time Fourier Transform (STFT) [2] (Fig. 2d) is performed in stage 6 . This technique is more effective for newborn palmprint image enhancement than Gabor filtering [4] that is based on the use of contextual filters whose parameters depend on the local ridge frequency and orientation. The STFT is able to simultaneously yield more robust local ridge orientation and ridge frequency information through a probabilistic approach. However, to further increase the image quality, we apply the Gabor filtering, using the most robust ridge orientation and ridge frequency information obtained after performing STFT, resulting in more well-defined ridges (Fig. 3d). An approach to Gabor filtering improvement, using more robust ridge orientation and ridge frequency information, is also proposed in [5].

Stages 7, 8 and 10 of our approach are the same as the ones presented in [4]. In stages 7 and 8, the image is divided into a set of $16 \times 16$ non-overlapping blocks to extract local information [4]. The local ridge orientation is defined for each block (Fig. 2e) and a window oriented along a direction normal to the local ridge orientation is defined for each block to estimate the local ridge frequency.

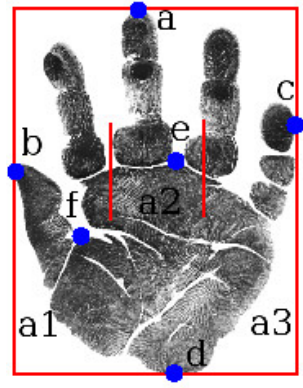

(a)

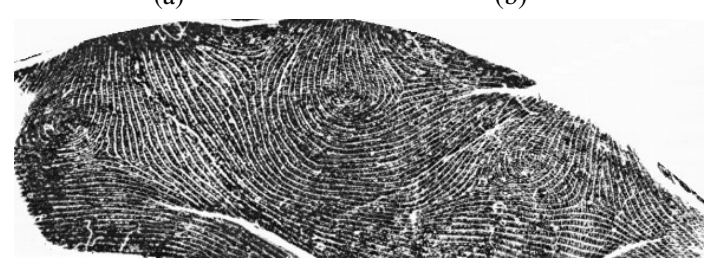

(c)

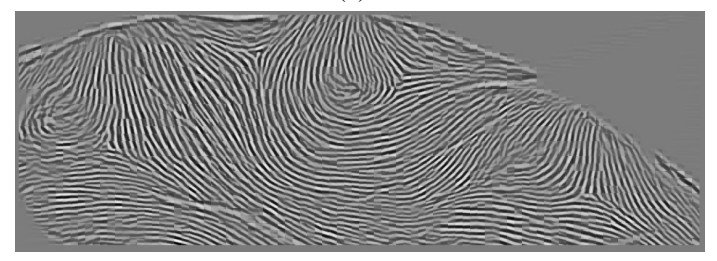

(d)

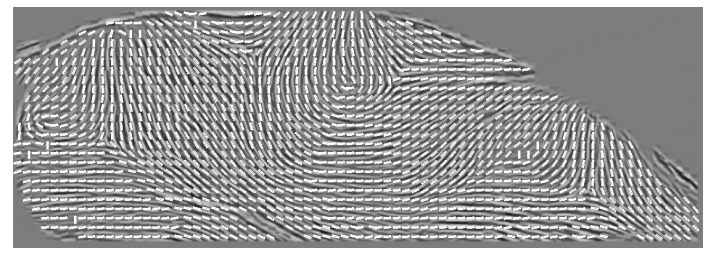

(e)

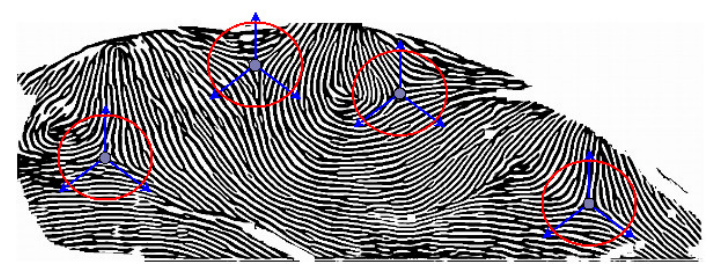

(f)

Figure 2. Image processing stages: (a) MBR, (b) finger removal, (c) region of interest, (d) STFT, (e) DF, (f) delta points.

In stage 9, the image is classified into two categories: recoverable and unrecoverable regions. Firstly, a global Otsu threshold (GOT) is calculated according to [8]. In addition, the image is divided into a set of $5 \times 5$ non-overlapping blocks to extract local information [4]. The recoverable blocks are identified to avoid false ridges or minutiae in the background or in very noisy/spoiled areas. The recoverable blocks correspond to the white area in Figs. $4 \mathrm{~b}$ and $4 \mathrm{~d}$. To detect these recoverable blocks, the gray level variance is locally computed for each block centered at pixel $(\mathrm{x}, \mathrm{y})$ 


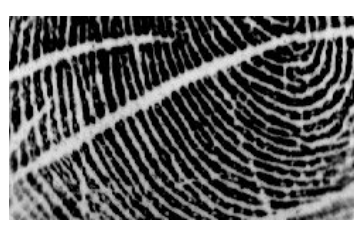

(a)

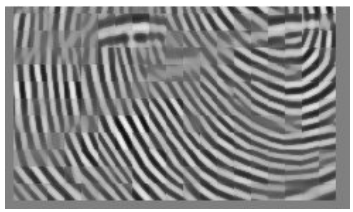

(c)

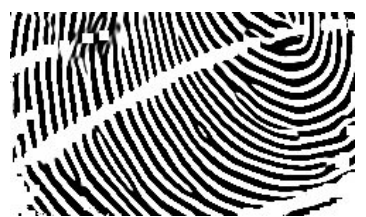

(b)

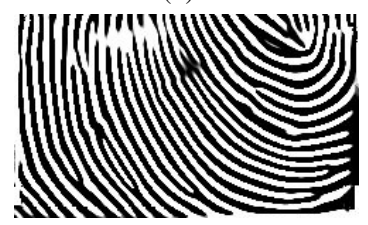

(d)
Figure 3. Enhancement process: (a) a region of the original image; (b) Gabor filtering; (c) STFT; (d) Gabor + STFT.

and oriented along $\mathrm{DF}[\mathrm{x}, \mathrm{y}]$, using the following equation:

$$
V_{i}=\frac{1}{n_{i}}\left(\sum_{j=1}^{n_{i}}\left(F_{i j}-M_{i}\right)^{2}\right),
$$

where $F_{i j}$ is the gray level value of pixel $j$ in block $i, n_{i}$ is the number of pixels in block $i$ and $M_{i}$ is the mean of the pixel values in block $i$.

A global gray level variance of the image is estimated using these local values according to the following equation:

$$
V_{G}=\frac{1}{n_{b}}\left(\sum_{i=1}^{n_{b}} V_{i}\right)
$$

where $n_{b}$ is the number of blocks in the image.

The blocks with variance values $\left(V_{i}\right)$ higher than the average of global variance and Otsu threshold $\left(\left(V_{G}+\mathrm{GOT}\right) / 2\right)$ are selected as recoverable. This is because usually fingerprint area presents higher variance than the background or noisy/spoiled areas.

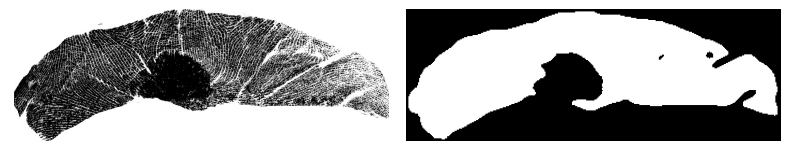

(a)

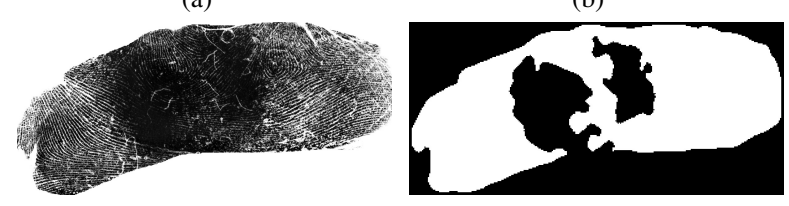

(c)

(d)

Figure 4. Recoverable area detection process: (a) and (c) Original images, (b) and (d) Recoverable areas in white.

The Gabor filtering in stage 10 improves the visibility of ridges structure in recoverable regions to generate an enhanced image (Fig. 2f). Finally, in stage 11, the PoincareIndex algorithm [1] extracts the delta points (Fig. 2f).
Stages 2 and 11 are very important to improve the performance of palmprint matching by reducing the orientation and translation differences between two palmprints.

\section{Matching Algorithm}

We propose a hybrid approach that combines two different strategies based on image registration using Simulated Annealing (SA) [7] and oriented texture fields (Finger-Code - FC) [6]. The hybrid approach has two stages. In the first stage, we look for the best matches of the input image in the gallery using the FC. The SA-based matcher then verifies if one of these matches is the best.

To create the authentication template, a thinning algorithm is performed on the enhanced image to generate a ridge map in order to extract the minutiae, as in [4]. The template includes $\left(n+C_{(n, 2)}\right)$ sub-templates (i.e., reference points), where $n$ is the number of delta points detected and $C_{(n, 2)}$ is the number of possible combinations of $n$ delta points to choose from; we set $n$ to 2 (no repetition; the order does not matter). We call these $C_{(n, 2)}$ sub-templates as Middle Regions (MR), i.e., the average coordinates between two delta points.

The sub-templates only include the information inside a ROI with a radius of 200 pixels around each one. Fig. $2 \mathrm{f}$ shows only the ROI around the delta points. Each subtemplate includes all ridge points (coordinates, orientation (DF) and radial distance to the reference point), minutiae (coordinates, orientation, radial distance to the reference point, number of other minutiae inside a region with a radius of 20 pixels around the correspondent minutia), and reference point (coordinates and orientation). In addition, 8 oriented components $\left(0^{\circ}, 22.5^{\circ}, 45^{\circ}, 67.5^{\circ}, 90^{\circ}, 112.5^{\circ}, 135^{\circ}\right.$ and $157.5^{\circ}$ ) are extracted using a directional bank of Gabor filters on the ROI tessellated with respect to the reference point (five concentric bands segmented into sixteen sectors $(5 \times 16=80$ sectors) $)$, generating a feature vector [6] (eight oriented components $(8 \times 80=640$ features $))$.

In the $\mathrm{FC}$ approach, the palm is divided into four regions of equal size. Theoretically, there is a delta point in a palmprint below each finger, i.e. four interdigital regions [3]. In order to calculate the similarity between two images, the matching (Euclidean distance) is performed only between feature vectors within the same region and of same type (delta point or middle region), resulting in $\left(n+C_{(n, 2)}\right)$ possible tests. Among these $\left(n+C_{(n, 2)}\right)$ resulting scores, the lowest one is selected.

In the SA-based matching approach, the palm is also divided into four regions and we only perform the matching between sub-templates within the same region and of the same type. Among the possible results, the matching that generates the highest similarity is selected. The verification stage performs the registration between ridges and minutiae by using a SA-based search technique [7]. Starting 
from a coarse alignment obtained by registering the reference points (Fig. 5a), the SA analyzes neighboring matches to look for a better alignment, which is computed as the number of overlapping points with the same direction. Two corresponding points are considered to be overlapping if they satisfy the following properties: (I) they must be inside a bounding circle of radius $r_{P}=1$ (Fig. 6); (II) they must have similar directions, i.e., the difference between the orientations $\left(\theta_{1}\right.$ and $\left.\theta_{2}\right)$ must be smaller than $\theta=10^{\circ}$; and (III) they must have similar radial distance, i.e., the difference between the radial distance $\left(\mathrm{rad}_{1}\right.$ and $\left.\mathrm{rad}_{2}\right)$ must be smaller than rad_dist $=10$. These thresholds were empirically selected based on the images in our database. Fig. 5 shows examples of the registration result using the proposed approach, where one can see the pre-alignment efficiency (Fig. 5a), the final alignment precision (Fig. 5b), a false matching (Fig. 5c) and true matching (Fig. 5d).

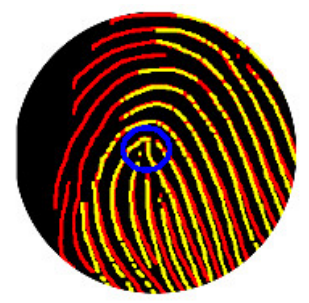

(a)

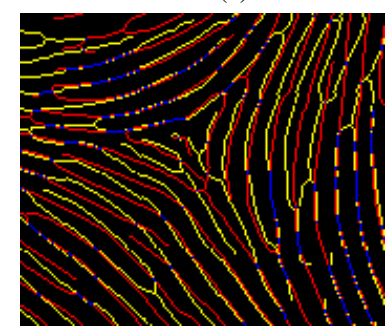

(c)

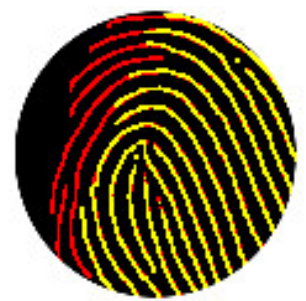

(b)

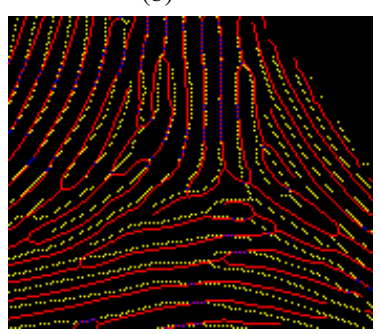

(d)
Figure 5. Registration process: (a) pre-alignment, (b) final alignment, (c) false matching and (d) true matching.

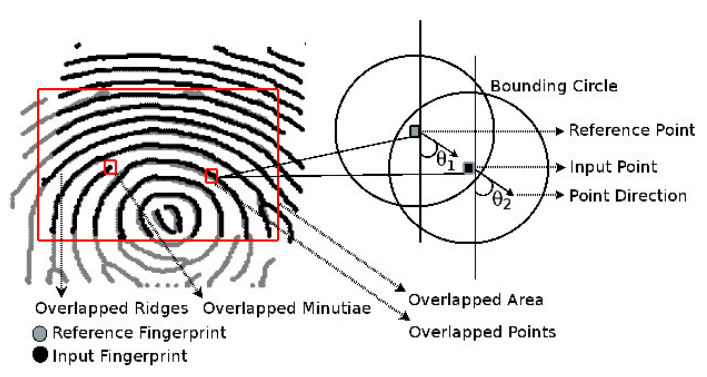

Figure 6. Registration process: overlapping area.

After registration, the similarity between two subtemplates is defined as a combination of the number of overlapping points with the same direction $S_{R}$ and the number of overlapping minutiae $S_{M}$. Two corresponding minutiae

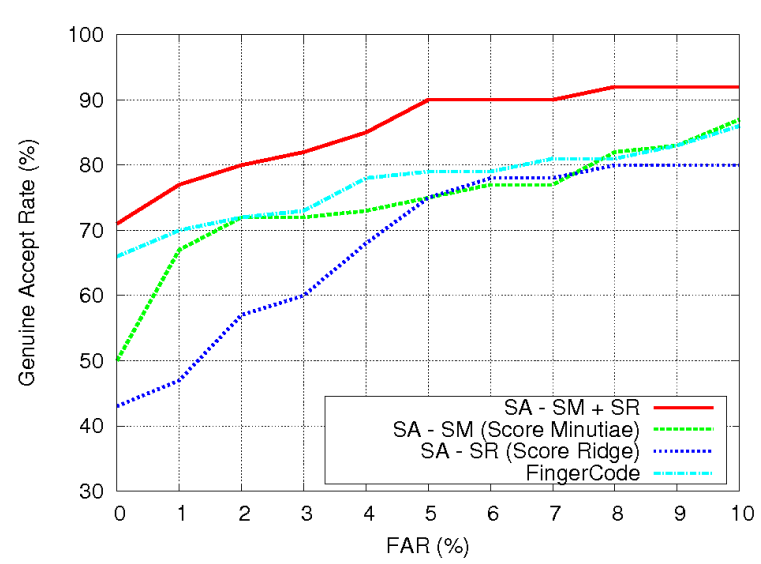

Figure 7. Recognition Results.

are considered to be overlapping if they satisfy the following properties: (I) they must be inside a bounding circle of radius $r_{M}=10$; (II) they must have similar directions, i.e., the difference between the orientations $\left(\theta_{1}\right.$ and $\left.\theta_{2}\right)$ must be smaller than $\theta=10^{\circ}$; (III) they must have similar radial distance, i.e., the difference between the radial distance $\left(\operatorname{rad}_{1}\right.$ and $\left.\mathrm{rad}_{2}\right)$ must be smaller than rad_dist $_{2}=10$; and (IV) they must have similar number of other minutiae around them, i.e., the difference between the number of other minutiae $\left(n M i n_{1}\right.$ and $n M i n_{2}$ ) must be smaller than num_min $=3$. These thresholds also were empirically selected.

The two matching scores $\left(S_{R}\right.$ and $\left.S_{M}\right)$ are combined to form the final score $S_{R+M}=\alpha_{1} S_{M}+\alpha_{2} S_{R}$, where $\alpha_{1}$ and $\alpha_{2} \in[0,0 ; 1,0]$. Fig. 7 shows the SA results using the best $\alpha_{1}$ and $\alpha_{2}$ values (SA $-0.66 * S_{M}+0.18 * S_{R}$ ), $S_{M}$ and $S_{R}$ results and the FingerCode results.

\section{Experimental Results}

To test the performance of the proposed approach, experiments were conducted on the NB_ID database. However, due to image quality problems, only palmprint images from 20 newborns could be used. These palmprints have sufficient ridge/minutiae information for recognition. We selected the three best palmprints from each one of the 20 newborns, totaling 60 palmprints. To verify the viability of automatic identification for these 20 newborns, we performed verification and identification experiments (see Table 1) based on retrieval rank and FAR critera [1].

It is important to report that in order to obtain better recognition rates, we must have visible ridges structure, minutiae and delta points. Unfortunately, many images, even those classified as good, using the image quality measure, do not meet these requirements. Some images present only one, two or three visible delta points, although they all should have four. We believe this problem mainly occurs due to the 1000ppi sensor resolution of Crossmatch used to 
capture the newborns' dactyloscopic features.

The delta points were chosen based on automatic analysis of the number of core and delta points detected in samples of the 20 babies. In most cases there was either no or only one core point. Moreover, in more than $80 \%$ samples three or more delta points were detected. However, another problem occurs when using delta points instead of core points: the ridges and oriented components around the delta points tend to form structures that are very similar among different individuals (as shown in Fig. 5c), adversely affecting both the recognition algorithms. As a result, we also decided to use the regions between two neighboring delta points (middle regions or MR). Figure 8 shows the improved results obtained using delta points (DP) and MR compared to the results obtained using only DP or MR.

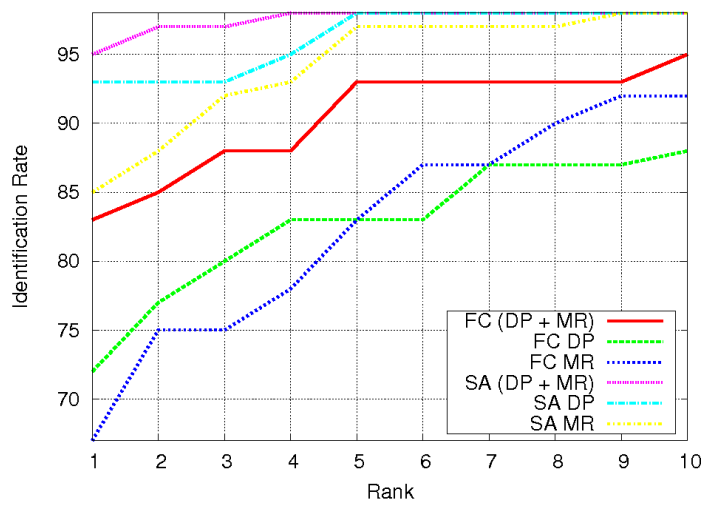

Figure 8. Identification Results.

As shown in Fig. 8, the SA approach gives the best results in both experiments. However, this approach is computationally costly compared to the FC method. We, therefore, decided to use FC for filtering the database and the SA only for verification purposes. The FC looks for the five best matches (Rank 5) of the input sample. Then the SA attests if one of these matches belongs to the subject associated with the input sample. Considering Rank 5 for FC and $0 \%$ FAR for SA $(0 \%$ FAR ensures that the SA will only accept true mates), we obtained $78 \%$ correct recognition rate. The hybrid approach improves the genuine accept rate when compared to the results of FC or SA methods individually.

\section{Summary}

This paper presents preliminary results on using 1000ppi dactyloscopic images for automatic newborn identification. Initial experiments on palmprints of 20 newborns show that the automatic identification process is fully executable, although difficult. To further improve the recognition performance, two main requirements are necessary: (1) the palmprint images of the newborns' dactyloscopic characteristics must have a higher resolution ( $>1500 \mathrm{ppi})$, and
(2) an appropriate protocol must be followed for acquiring the images (e.g., cleaning the palmar surface and applying the right pressure between the palm and the sensor). However, it is important to point out that there is no commercially available equipment as of now that is capable of satisfying this application requirement. Despite the limitations of the CrossMatch sensor, it was able to acquire palmprints images with sufficient quality for newborn identification for some of the newborns. Compared to DNA testing, the proposed approach is acceptable for newborn identification since it is non invasive. Using the knowledge gained so far on this project, we have started a new phase of image acquisition in one of the largest maternity hospitals in $\mathrm{Cu}$ ritiba. In this new protocol, we aim to collect good quality images of at least 100 babies and their mothers' fingerprints. In order to collect these images, we are using the best image acquisition protocol developed in this research.

\section{References}

[1] R. M. Bolle, J. H. Connell, S. Pankanti, N. K. Ratha, and A. W. Senior. Guide to Biometrics. Springer, 2003.

[2] S. Chikkerur, A. N. Cartwright, and V. Govindaraju. Fingerprint enhancement using STFT analysis. Pattern Recognition, 40(1):198-211, 2007.

[3] H. Cummins and C. Midlo. Finger prints, palms and soles. New York: Dover Publications., 1943.

[4] L. Hong, Y. Wan, and A. K. Jain. Fingerprint image enhancement: Algorithm and performance evaluation. IEEE Trans. on PAMI, 20(8):777-789, 1998.

[5] A. K. Jain and J. Feng. Latent palmprint matching. IEEE Trans. on PAMI, 31(6):1032-1047, 2009.

[6] A. K. Jain, S. Prabhakar, L. Hong, and S. Pankanti. Filterbank-based fingerprint matching. IEEE TIP, 9(5):846859, 2000.

[7] S. Kirkpatrick, C. Gelatt, and M. Vecchi. Optimization by simulated annealing. Science, 220:671-680, 1983.

[8] N. Otsu. A threshold selection method from gray-level histograms. IEEE Trans. on Systems, Man, and Cybernetics, 9(1):919-926, 1979.

[9] A. Ross, K. Nandakumar, and A. K. Jain. Handbook of Multibiometrics. Springer, 2006.

[10] L. Silva, O. R. P. Bellon, R. P. Lemes, J. A. Meira, and M. N. L. Cat. An image processing tool to support gestational age determination. In Proc. IEEE CBMS, pages 867874, 2006.

[11] D. Weingaertner, O. R. P. Bellon, L. Silva, and M. N. L. Cat. Newborn's biometric identification: can it be done? International Conference on Computer Vision Theory and Application (VISAPP), pages 200-205, 2008.

[12] C. Wu, S. Tulyakov, and V. Govindaraju. Image quality measures for fingerprint image enhancement. Lecture Notes in Computer Science, 4105:215-222, 2006.

[13] K. Zuiderveld. Contrast Limited Adaptive Histogram Equalization. Graphics Gems IV, pages 474-485. Academic Press Professional, 1994. 